$63^{\text {ème }}$ Congrès de la SFCO, 03033 (2015)

DOI:10.1051/sfco/20156303033

(C) Owned by the authors, published by EDP Sciences, 2015

\title{
Manifestation gingivale d'un myélome multiple connu. À propos d'un cas
}

\author{
Abbas A, Canonica M, Limam A, Dauzat A, Lesclous P, Kimakhe S \\ Unité fonctionnelle de Chirurgie orale, service d'Odontologie Restauratrice et chirurgicale, CHU de Nantes, \\ 1 place Alexis-Ricordeau, 44000 Nantes
}

Introduction

Le myélome multiple (MM) ou maladie de Kahler est une hémopathie maligne caractérisée par une prolifération plasmocytaire monoclonale, anarchique et tumorale, envahissant la moelle osseuse hématopoïétique. L'atteinte des structures maxillo-faciales dans l'évolution du MM est assez fréquente, notamment au niveau du maxillaire et de la mandibule, et représente parfois la circonstance de découverte de la maladie. Pour certains auteurs, la localisation maxillaire est la plus fréquente (1), alors que pour d'autres c'est la mandibule, dans sa partie postéro-angulaire, qui est la plus touchée (2). Observations

Une patiente âgée de 69 ans s'était présentée en avril 2014 pour prise en charge de tuméfactions gingivales douloureuses apparues 3 semaines auparavant et entraînant une gêne fonctionnelle à l'alimentation. Elle était atteinte d'un myélome multiple à IgG lambda, à un stade avancé (stade 3), diagnostiqué en 2011. L'examen clinique montrait des lésions hypertrophiques gingivales en regard des 16, 36 et 37. Elles étaient de couleur violacée, leur état de surface était lisse, leur consistance molle, leurs limites mal définies. Elles étaient adhérentes aux structures périphériques, et douloureuses à la palpation (EVA 70). La radiographie panoramique révélait à ce niveau des images d'ostéolyse plus ou moins prononcées, diffuses, sans résorption radiculaire associée. La NFS révélait une bicytopénie avec une leucopénie à 3,80 Giga/l, et une anémie normocytaire à $11,3 \mathrm{~g} / \mathrm{dl}$. Devant l'absence d'éléments en faveur d'une étiologie locale, une origine plasmocytaire était fortement suspectée. Une biopsie a donc été réalisée. L'examen histologique qui a suivi confirmait le diagnostic de prolifération plasmocytaire gingivale. Il fut alors décidé en réunion de concertation pluridisciplinaire de réaliser une chimiothérapie (association agent alkylant anti-tumoral et cytocide/ glucocorticoïde). Devant le peu d'efficacité, pendant 5 mois, de cette association, l'agent alkylant a été substitué par un immuno-modulateur de dernière génération: le pomalidomide. Malgré cela, la patiente décédait 2 mois plus tard.

Commentaire

Le pronostic vital des patients atteints de ce type de pathologie est souvent engagé et il est corrélé à la précocité du diagnostic. L'atteinte orale n'étant pas négligeable, le chirurgien-dentiste est donc le praticien le mieux placé pour évoquer ce type de diagnostic. Cela nécessite une démarche rigoureuse : examen clinique, imagerie (panoramique dentaire, clichés rétro-alvéolaires voire CBCT), et réalisation d'une biopsie à des fins de caractérisation histologique.

Le patient sera, en cas de confirmation, orienté vers une structure spécialisée dans la prise en charge globale de ce type de pathologie ; généralement un service d’hématologie.

Conclusion

Ce cas clinique nous rappelle l'importance du rôle du chirurgien-dentiste dans la détection et le dépistage précoce des lésions suspectes au niveau de la cavité orale. 


\section{Références}

(1) Lae et al. (2003)

(2) Lambertenghi-Deliliers et al. (1988)

Nom et adresse du conférencier

Amine ABBAS

Unité fonctionnelle de Chirurgie orale, service d'Odontologie restauratrice et chirurgicale CHU de Nantes

1 place Alexis-Ricordeau

44000 Nantes (France)

amine.abbas@univ-nantes.fr 